\title{
A Web-Based Multimedia Diabetes Mellitus Education Tool for School Nurses
}

\author{
Zhongfei (Mark) Zhang \\ Computer Science Dept. \\ Watson School \\ State Univerisity of New York \\ Binghamton, NY 13902 \\ zhongfei@cs. binghamton. edu
}

\author{
Paul E. Knudson \\ Ruth S. Weinstock \\ Suzanne Meyer \\ Joslin Diabetes Center \\ State University of New York \\ Upstate Medical University \\ 90 Presidential Plaza, Syracuse, $N Y 13202$ \\ \{knudsonp, weinstor, meyers $\}$ @upstate.edu
}

\begin{abstract}
This paper presents a Web based diabetes mellitus education program for school nurses, called WebDiamen. Compared with existing systems for medical education using computer-assisted technologies, WebDiamen exhibits the advantages of powerful browsing capability, friendly GUI, individualized presentation schedule tailored to each user based on their special browsing interest, powerful online processing and annotation capabilities, and powerful querying capabilities. The system is being evaluated for school nurses in Onondaga County, NY.
\end{abstract}

\section{Introduction}

Diabetes mellitus is a chronic metabolic disease. It can have devastating shortterm and long-term complications. With effort, diabetes mellitus complications can be prevented. Striving toward good control requires a team approach, consisting of the patient, diabetes nurse educators, dietitian, primary care physician, diabetes physician specialists, and social support. In school age children, the school nurse needs to be integrated into the team. The school nurse should provide support, accurate information, and re-enforce good self-management skills. The school nurse needs a level of comfort and expertise to meet this role. We propose to develop a web-based tool that will provide multimedia and multimodal information to the school nurse to be accessed when the information is needed, improving the school nurse' effectiveness.

To meet this educational objective, we have designed a multimedia database system that is accessed over the web. The proposed application scenario is that a school nurse can access to this system using a standard browser from her PC at any place at any time to update her knowledge about diabetes mellitus, and to share or exchange information in a timely fashion with her colleagues through the Internet. Future implementation can use the same technology to inform other health professionals, those with diabetes mellitus and their families. 
Diabetes education is important in improving diabetes self-management and providing effective diabetes treatment in healthcare communities. Brown [3] reviewed diabetes education literature over the past 20 years, and Glasgow [5] and Walker [15] recently discussed and proposed several strategies for improving diabetes education. In particular, management of children with diabetes has received focused attention by healthcare providers as well as other healthcare professionals [9]. Siminerio and Koerbel [13], for example, reported a community program called "5 Cs of diabetes", which had the aim of raising awareness of and improving diabetes education for school personnel.

The use of computer-assisted technologies in diabetes as well as other medical domain education has been a focus of research in recent years by experts in medical informatics, medical education, as well as other specific medical areas. This has been possible because of the rapid development of computer technologies, especially the development of Internet technologies and multimedia database and network tech-

nologies. Zucker et al [16] was one of the first to develop web-based multimedia applications in medical education. More recent developments may be found in the work of Murray and Anthony [8] and Grimson et al [6]. Shegog et al developed a computer-assisted asthma self-management educational program loaded in a CDROM called Watch, Discover, Think, and Act (WDTA) which addressed behaviorial and motivational issues for urban and minority children ages 9 to 13 [12]. Feil et al [4] studied the feasibility of the use of computer-assisted technologies for medical education in patients with almost no computer literacy.

Most of the existing commercial and research systems for multimedia based medical education, especially diabetes education, only allow users to browse the system through mouse clicking on a GUI, and/or through key word based querying to access information from the system. These systems either do not have a database, or only have a "dead" database, i.e., they do not allow users to change the database. In contrast, our system has a live database which allows users to "contribute" their experiences or comments as well as any case studies into the database, and consequently allows users to share information with their colleagues in the community.

In this paper, a prototype system is presented for this new paradigm of Web based diabetes mellitus education program for school nurses. This prototype system is called WebDiamen. As compared with existing technologies described in the literature, our system has a more powerful browsing capability, and a more friendly GUI. In addition, WebDiamen has the following three unique features: (i) powerful online processing and online annotatoin capabilities (ii) an individualized presentation schedule tailored to each user based on their personal browsing interest (iii) powerful querying capabilities. This paper presents an overview of the technological features of WebDiamen. The next section briefly reviews the system architecture of WebDiamen. This is followed by a discussion of the currently implemented functionalities of WebDiamen. Lastly a section briefly discussing the future testing and evaluation of WebDiamen is presented.

\section{System architecture}

The WebDiamen architecture is based on the standard client-server model with 
a 3-tier structure. The backbone server uses a Microsoft SQL Server installed in a dedicated Dell XPS $800 \mathrm{MHz}$ Pentium III with 40GB disk space. The middleware server is implemented in a dedicated Dell XPS $866 \mathrm{MHz}$ Pentium III with 40 GB disk space. The client side may be any computer running any operating system connected to the Internet. The architecture is implemented in Java. At the client side, a user runs a Java applet in a standard browser window to access to the system. The user must have password-protected membership. The communication between the client machine and the middleware is achieved by Remote Method Invocation (RMI) and the applet running at the client machine and a servlet running at the middleware. The communication between the middleware and the backbone database is achieved by MS SQL Server supported query and retrieval functionalities.

\section{$3 \quad$ Implemented functionalities}

There are two types of users defined. An instructor user can access to all the data in the system, process the data, make annotations to the data, and upload exam questions/answers and/or processed/annotated data into the system. A student user can only browse the information, including reading and/or viewing/listening to the lectures, taking exams, checking the solutions, posing questions, etc., but is not allowed to construct exam questions or make annotations to the data or process the data. Both types of users are provided with the functionalities of searching for specific material and/or making comments on particular pieces of data at anytime during their browsing.

In our WebDiamen application, the school nurses will be the student users and will sign up for the free membership online; the instructor will be the clinical staff from the Joslin Diabetes Center. After membership logins, the main page shows six different major options for browsing.

View lectures: A user clicking this pointer can access to different subjects related to diabetes mellitus education; clicking each of the subjects leads to different presentations sorted by days (e.g., day 1, day 2, etc.); finally, clicking a specific day's topic leads to classes for different media types for the presentation, such as powerpoint slides, video recordings, textual documents, audio recordings, or any of the above in combination. When the user chooses one of the media types, a window pops up for this specific presentation in the chosen media.

Take exams: This pointer leads to a menu of different subjects, and when a specific subject is chosen, all the available exams for this subject are listed. When the user chooses a specific exam, a window pops up showing questions of this exam. A time counter pops up to measure the time the user spent on this exam, which is used in determining the user's grade of the exam.

Guidelines: This provides links to well-established diabetes mellitus education sites. Currently, websites of NYS Department of Health children diabetes education and management and American Diabetes Association are linked.

Chat room: This leads to a chat room window for real-time, online dialog.

Online processing: A set of functionalities is available under this pointer. These functionalities allow users to online process the information they are browsing, and allow them to save the processed data into their local machines, and/or to contribute 
to the database by uploading the information into it, such that this new information may be shared by other users. The implemented online processing capabilities include zooming, comparing, highlighting with different pseudo-colors, histogramming, thresholding, as well as composing a new image from other images, etc.

Online annotation: The unique annotation functionality allows users to online annotate the information they are browsing. This may be used along with any online processing capabilities, and the annotated data may be either saved to users' local machine, and/or contributed to the database by uploading the data into the system. This may help create a medium to allow users to share their individual experience or as a way of expressing their feedback after reviewing a specific piece of information in the database. This permits live communications and data sharing, and consequently can lead to a live and rich database as opposed to a "dead" database, as all the annotations are indexed.

In addition to the six "major" functions described above, at any point of the user browsing, there are always two buttons available:

Bulletin board: This allows users to make comments at anyplace during their browsing, and the comments are indexed in the database. WebDiamen marks each comment as originating from a student or from an instructor. This allows an instructor to receive feedback from students effectively and efficiently, and also encourages interaction and exchange of information between users.

Query and search: WebDiamen allows much more powerful querying capabilities than exist in most systems. In addition to textual key word query, the system also allows image query, and a user may query the system using both of the modalities. For example, after having reviewed a specific diabetes presentation from the system, a school nurse may have a question related to an individual patient case, which she can document in a whole folder with both graphical and textual information. She can send the whole folder as a query to the system, and the system will return back to her with all the relevant information in response to the query.

In order to serve as an effective educational tool, one of the foci of the research leading to WebDiamen was to design a multifunctional and multimodal GUI to allow a user to do online browsing, querying, annotating, saving and uploading, and processing specific information of his/her interest, and to dynamically propose a presentation schedule to the user while he/she is accessing the system with different possible browsing patterns or different searching interests.

Essentially, there are two different patterns of presentation. If a user follows a specific browsing sequence, such as View lectures $-->$ Insulin and Insulin Delivery Systems --> Day $1-\rightarrow$ Powerpoint, the mode of presentation is not an issue, and only requires popping up a window to deliver the presentation. On the other hand, if a user enters a query Insulin Delivery Systems, there are numerous different possible modalities in which information is stored in the database related to this query. The design of a dynamic presentation schedule based on this user's individual browsing pattern and interest is a significant research issue addressed in this project. Possible modalities of presentations include textual documents, lecture or other video/audio clips, images, graphics/animation, Power-point, as well as possible combinations synchronized between two or even more of these modalities. Multimedia presentation or authoring is an open area in multimedia research with many proposed methodologies in the literature (e.g., [2]). In the current version of 
WebDiamen, the user's initial browsing history is tracked, and based on machine learning techniques [7], a best presentation schedule is determined and executed.

Given the many different modalities of data stored in the database, how to efficiently and effectively index them into the database is another research focus. Again, multimedia information indexing and retrieval is another open area in the literature [14]. In the current version of WebDiamen, we focus on two modalities: imagery and text. For text modality indexing, the standard inverted file indexing technique is used [11]. For imagery modality indexing, geometric histogram recently proposed by Rao et al [10] is employed. Developing more effective and efficient indexing methods for image data as well as for the other data modalities remain a major research objective in this project.

\section{Evaluation plan}

At this time of publication, the design and implementation of a prototype version of WebDiamen has been completed, and its evaluation has been initiated. The evaluation protocol for WebDiamen is designed as follows. The database is populated with multimedia diabetes mellitus educational materials in different modalities constructed by the staff at our Joslin Diabetes Center at SUNY Upstate Medical University, Syracuse, NY. The data will be authoritative, using well-established guidelines and information.

School nurses for this evaluation process will be recruited from throughout Onondaga County, NY. The school nurses will use a secure login, so that the nurses' use of the site will be tracked. The school nurses will be asked to visit and familiarize themselves with the web site of WebDiamen. During the initial visit to the web site, they will fill out an online survey. The survey will use Diabetes Attitude Scale 3. This instrument is a validated measure of general diabetes related attitudes [1]. The web site usability and user satisfaction will also be tracked.

An effort will be made to recruit as many school nurses with Internet access in Onondaga County as possible. The change in responses to the Diabetes Attitude Scale 3, user satisfaction, and use of the system by nurses overtime will be compared and will be reported in future publications.

\section{Conclusion}

A diabetes mellitus educational system for school nurses called WebDiamen is presented, which employs state-of-the-art technology in multimedia database research, and allows school nurses to access to the system at any place at any time for the purpose of diabetes mellitus education and self-management training. This system is currently being evaluated. After the initial evaluation phase using school nurses from Onondaga County, NY, WebDiamen will be applied and studied more widely, including use by other health care providers, those who care for people with diabetes and those who have diabetes mellitus. 


\section{Acknowledgements}

This research is supported in part by SUNY Binghamton through faculty startup funds, and in part by the NYS Department of Health and the SUNY Upstate Medical University, Syracuse, NY. We thank Ruofei Zhang, Jie Zhou, and Jay Parikh for their assistance with the implementation of the system.

\section{References}

[1] R.M. Anderson, J.T. Fitzgerald, M.M. Funnell, and L.D. Gruppen. The third version of the diabetes attitude scale. Diabetes Care, 21:1403-1407, 1998.

[2] R. Baecker, A.J. Rosenthal, N. Friedlander, E. Smith, and A. Cohen. A multimedia system for authoring motion pictures. In Proc. ACM Multimedia, 1996.

[3] S.A. Brown. Interventions to promote diabetes self-management: state of the science. The diabetes Educator, 25(6 (Suppl)):52-61, 1999.

[4] E.G. Feil, R.E. Glasgow, S. Boles, and H.G. McKay. Who participates in internet-based selfmanagement programs? a study among novice computer users in a primary care setting. The Diabetes Educator, 26(5):806-811, 2000.

[5] R.E. Glasgow. Outcomes of and for diabetes education research. The Diabetes Educator, 25(6 (Suppl)):74-88, 1999.

[6] J. Grimson, W. Grimson, M. Flahive, C. Foley, R. O'Moore, J. Nolan, and G. Chadwick. A multimedia approach to raising awareness of information and communications technology amongst healthcare professionals. International Journal of Medical Informatics, 58-59:297305,2000 .

[7] T. Mitchell. Machine Learning, McGraw Hill, 1997.

[8] P.J. Murray and D.M. Anthony. Current and future models for nursing e-journals: making the most of the Web's potential. International Journal of Medical Informatics, 53(2-3):151-161, 1999.

[9] American Association of Diabetes Educators. Management of children with diabetes in the school setting. The Diabetes Educator, 25(6):873-877, 1999.

[10] A. Rao, R. Srihari, and Z. Zhang. Geometric histograms: a distribution of geometric configurations of color subsets. In Proc. SPIE International Conference on Internet Imaging. SPIE, 2000 .

[11] G. Salton. Automatic text processing: the transformation, analysis, and retrieval of information by computer. Addison Wesley, 1989.

[12] R. Shegog, L.K. Bartholomew, G.S. Parcel, M.M. Sockrider, L. Masse, and S.L. Abramson. Impact of a computer-assisted education program of factors related to asthma selfmanagement behavior. Journal of American Medical Informatics Association, 8(1):49-61, 2001.

[13] L.M. Siminerio and G. Koerbel. A diabetes education program for school personnel. Practical Diabetes Int, 17(6):174-177, 2000.

[14] R.K. Srihari, Z. Zhang, and A. Rao. Intelligent indexing and semantic retrieval of multimodal documents. Information Retrieval, Special Issue on Document Image Retrieval, 2(2/3):245-275, May 2000.

[15] E.A. Walker. Characteristics of the adult learner. The Diabetes Educator, 25(6 (Suppl)):16-24, 1999.

[16] J. Zucker, H. Chase, P. Molholt, C. Bean, and R.M. Kahn. A comprehensive strategy for designing a Web-based medical curriculum. In Proc. AMIA Annual Fall Symposium, pages $41-45,1996$. 